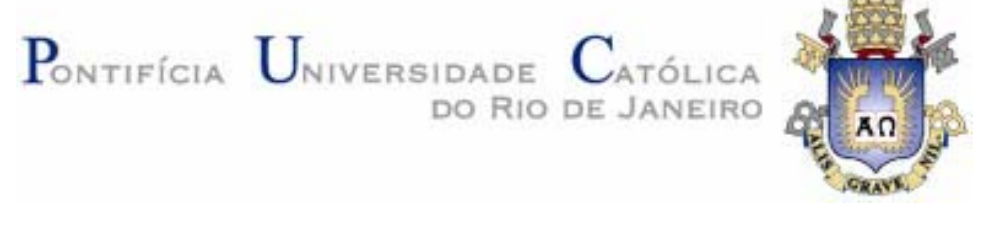

Pablo Motta Ribeiro

\title{
Remuneração dos Serviços Ancilares de Suporte de Potência Reativa e Reserva de Potência Quando Providos por Geradores
}

Dissertação de Mestrado

Dissertação apresentada como requisito parcial para obtenção do título de Mestre pelo Programa de PósGraduação em Engenharia Elétrica da PUC-Rio.

Orientadores: Ricardo Bernardo Prada Albert Cordeiro Geber de Melo 

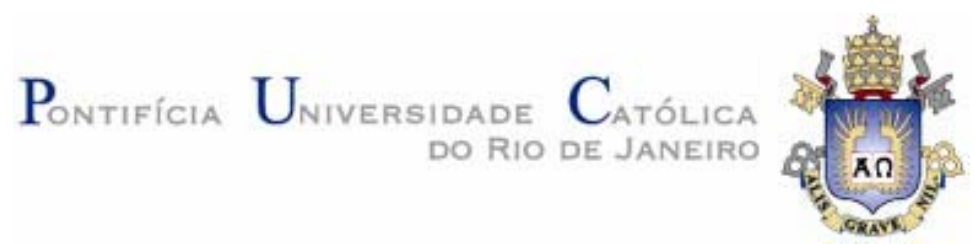

Pablo Motta Ribeiro

\section{Remuneração dos Serviços Ancilares de Suporte de Potência Reativa e Reserva de Potência Quando Providos por Geradores}

Dissertação de Mestrado apresentada como requisito parcial para obtenção do título de Mestre pelo Programa de PósGraduação em Engenharia Elétrica da PUC-Rio. Aprovada pela Comissão Examinadora abaixo assinada.

Prof. Dr. Ricardo Bernardo Prada Orientador DEE/PUC-Rio

Prof. Dr. Albert Cordeiro Geber de Melo Co-Orientador CEPEL / IME / UERJ

Dr. Luiz Guilherme Barbosa Marzano CEPEL

Dr. João Ricardo Paes de Barros CHESF

Prof. José Eugênio Leal Coordenador(a) Setorial do Centro Técnico Científico - PUC-Rio

Rio de Janeiro, 01 de abril de 2005 
Todos os direitos reservados. É proibida a reprodução total ou parcial do trabalho sem autorização da universidade, do autor e do orientador.

Pablo Motta Ribeiro

Graduou-se em engenharia elétrica em 1999 pela Universidade Federal de Juiz de Fora. Atualmente trabalha como pesquisador no Centro de Pesquisas de Energia Elétrica - CEPEL.

Ficha Catalográfica

Ribeiro, Pablo Motta

Remuneração dos serviços ancilares de reserva de potência e suporte de potência reativa quando providos por geradores / Pablo Motta Ribeiro ; orientador: Ricardo Bernardo Prada. - Rio de Janeiro : PUC-Rio, Departamento de Engenharia Elétrica, 2004.

$144 \mathrm{f.} ; 30 \mathrm{~cm}$

Dissertação (mestrado) - Pontifícia Universidade Católica do Rio de Janeiro, Departamento de Engenharia Elétrica.

Incluí referências bibliográficas.

1. Engenharia Elétrica - Teses. 2. Serviços ancilares. 3. Suporte de potência reativa. 4. Reserva de potência. 5. Teoria dos jogos cooperativos. 6. Fluxo de potência ótimo. 7. Confiabilidade composta. I. Prada, Ricardo Bernardo. II. Pontifícia Universidade Católica do Rio de Janeiro. Departamento de Engenharia Elétrica. III. Título.

CDD: 621.3 
À minha esposa, Marcia, e sua filha, Andressa Aos meus pais, Jorge e Cida À minha irmã, Paloma 


\section{Agradecimentos}

Aos professores e orientadores Ricardo Prada e Albert, pela orientação e conhecimentos partilhados ao longo deste trabalho.

À Marcia e Andressa, pela paciência e compreensão nos momentos difíceis.

Aos amigos Luiz Guilherme e Javier, pela assistência e apoio no desenvolvimento deste trabalho.

Aos amigos do CEPEL, que sempre me apoiaram ao longo deste trabalho.

Aos amigos da PUC, pelo convívio agradável e pelo apoio técnico recebido.

Ao Centro de Pesquisas em Energia Elétrica, pela disponibilização do tempo e das ferramentas computacionais que propiciaram a conclusão deste trabalho.

À Pontifícia Universidade Católica, pelo suporte técnico e financeiro. 


\section{Resumo}

Ribeiro, Pablo Motta. Remuneração dos Serviços Ancilares de Suporte de Potência Reativa e Reserva de Potência Quando Providos por Geradores. Rio de Janeiro, 2005. 144p. Dissertação de Mestrado Departamento de Engenharia Elétrica, Pontifícia Universidade Católica do Rio de Janeiro.

Este trabalho apresenta metodologias para definir a remuneração dos geradores que provêem serviços ancilares em função do benefício proporcionado ao sistema. São analisados os serviços ancilares de suporte de potência reativa e reserva de potência. Para o serviço ancilar de suporte de potência reativa, o valor do benefício proporcionado pelos geradores é obtido por meio do custo evitado de alocação de novas fontes de potência reativa. Já para o serviço ancilar de reserva de potência, o benefício é calculado a partir da redução observada no valor esperado de energia não suprida, multiplicada pelo custo unitário de interrupção do sistema de potência. $O$ valor de benefício proporcionado por cada serviço ancilar é determinado por meio de um algoritmo de pontos interiores em um problema de fluxo de potência ótimo. A remuneração que cabe a cada gerador é definida por meio do emprego do método de repartição de custos de Aumann-Shapley, oriundo da Teoria dos Jogos Cooperativos. A aplicação deste método garante que a repartição do benefício entre os geradores seja realizada de forma justa e eficiente. As metodologias propostas para são ilustradas em dois sistemas de potência distintos: o sistema IEEE-RTS e o sistema Sul-Sudeste Brasileiro. Os resultados obtidos são discutidos e a eficácia da metodologia é analisada.

\section{Palavras-chave}

serviços ancilares; reserva de potência; suporte de potência reativa; teoria dos jogos cooperativos; fluxo de potência ótimo; confiabilidade composta 


\section{Abstract}

Ribeiro, Pablo Motta. Pricing the Ancillary Services of Reactive Power Supply and Generation Reserve When Provided by Generators. Rio de Janeiro, 2005. 144p. M.Sc. Dissertation - Electrical Engineering Department, Pontifícia Universidade Católica do Rio de Janeiro.

This work presents methodologies to define the remuneration of the generators that provide ancillary services to the power system as a function of the benefit provided to the system. In this work the ancillary services considered are the reactive power supply and the generation reserve. To the ancillary service of reactive power supply, the benefit provided by the generators is calculated by the avoided cost of new reactive power sources. The benefit provided by the ancillary service of generation reserve is calculated as the reduction on the expected energy not supplied, multiplied by the interruption cost of the power system. An optimum power flow is used to determine the benefit provided by each ancillary service. The remuneration of each generator is defined through the cost allocation method of Aumann-Shapley, which belongs to the Cooperative Game Theory. The Aumann-Shapley method guarantees that the payment partition among the generators is fair and efficient. The proposed methodology is applied to the IEEE-RTS power system and the Brazilian South-Southwest power system. The numerical results are discussed and the efficiency of the methodology is analyzed.

\section{Key words}

ancillary services; generation reserve; reactive power supply; cooperative game theory; optimum power flow; composite reliability 


\section{Sumário}

1 Introdução 15

1.1. Considerações Gerais 15

1.2. Objetivo 16

1.3. Estado da Arte 17

1.4. Estrutura da Dissertação 21

2 Métodos de Repartição de Custos 23

2.1. Introdução 23

2.2. Conceitos Básicos 24

2.3. Exemplo llustrativo $\quad 28$

2.4. Método Nucleolus $\quad 30$

2.5. Método dos Custos Marginais 33

2.6. Método dos Custos Incrementais 36

2.7. Método de Shapley 38

2.8. Método de Shapley Modificado $\quad 39$

2.9. Método de Aumann-Shapley 41

2.10. Conclusões 44

3 O Problema de Fluxo de Potência Ótimo 46

3.1. Introdução 46

3.2. Fluxo de Potência $\quad 47$

3.2.1. Método de Newton-Raphson 49

3.3. Fluxo de Potência Ótimo $\quad 50$

3.3.1. Restrições de Igualdade $\quad 51$

3.3.2. Restrições de Desigualdade 53

3.3.3. Funções-objetivo $\quad 54$

3.4. Resolução do Problema do FPO 57

3.4.1. Método de Pontos Interiores 58

3.4.2. Condições de Otimalidade $\quad 59$

3.4.3. Resolução do Sistema de Equações 60

3.4.4. Passo Primal-Dual 62 
3.4.5. Atualização do Parâmetro Barreira 62

3.4.6. Algoritmo de Solução 63

3.5. A Função Valor Ótimo de um Problema de Otimização 64

3.6. Conclusões 65

4 Análise de Confiabilidade em Sistemas de Potência 67

4.1. Introdução $\quad 67$

4.2. Níveis Hierárquicos de Confiabilidade 68

4.3. Conceitos Gerais $\quad 69$

4.4. Definição de Algumas Funções-Teste 72

4.4.1. Função-Teste para LOLP 72

4.4.2. Função-Teste para EPNS 72

4.4.3. Função-Teste para LOLE 72

4.4.4. Função-Teste para EENS 73

4.5. Métodos de Seleção de Estados

4.5.1. Método de Enumeração de Estados $\quad 74$

4.5.2. Simulação de Monte Carlo $\quad 75$

4.5.3. Cálculo da Precisão das Estimativas 76

4.6. Análise de Desempenho

4.6.1. Etapa de Configuração de Estados $\quad 77$

4.6.2. Etapa de Pré-Solução 78

4.6.3. Etapa de Solução $\quad 78$

$\begin{array}{ll}\text { 4.6.4. Etapa de Pós-Solução } & 79\end{array}$

4.6.5. Etapa de Medidas Corretivas $\quad 79$

4.7. Algoritmo de Simulação Monte Carlo Não-Sequencial 79

4.8. Conclusões 80

5 Metodologia para Remuneração do Serviço Ancilar de Suporte de Potência Reativa Quando Provido por Geradores 82

5.1. Introdução 82

5.2. O Problema de Mínimo Custo de Instalação de Novas Fontes de Potência Reativa $\quad 84$

5.3. Aplicação do Método de Aumann-Shapley à Metodologia Proposta 86

5.4. Algoritmo de Solução

5.5. Exemplo Numérico $\quad 91$

5.6. Resultados Numéricos $\quad 97$

5.7. Conclusões 103 
6 Metodologia para Remuneração do Serviço Ancilar de Reserva de Potência Quando Provido por Geradores 105

6.1. Introdução 105

6.2. O Problema de Mínimo Custo de Corte de Carga 108

6.3. Aplicação do Método de Aumann-Shapley à Metodologia Proposta 110

6.4. Algoritmo de Solução 113

6.5. Exemplo Numérico 116

6.6. Resultados Numéricos 122

6.7. Conclusões 126

7 Conclusões e Propostas para Trabalhos Futuros 128

8 Referências Bibliográficas 131

9 Apêndice A - Método de Aumann-Shapley 135

10 Apêndice B - Dados de Entrada para o Sistema-Exemplo 139

11 Apêndice C - Resultados Numéricos para a Metodologia de Precificação do Serviço Ancilar de Suporte de Potência Reativa 141

12 Apêndice D - Resultados Numéricos para a Metodologia de Precificação do Serviço Ancilar de Reserva de Potência 144 


\section{Lista de figuras}

Figura 2-1 - Definição Gráfica do Núcleo 27

Figura 2-2 - Representação Geométrica do Núcleo 30

Figura 2-3 - Representação gráfica dos custos marginais 33

Figura 2-4 - Sobre-remuneração do método de custos marginais 35

Figura 2-5 - Custo Incremental do Serviço - Seqüência 1-2-3 37

Figura 2-6 - Custo Incremental do Serviço - Seqüência 1-3-2 38

Figura 2-7 - Número de Combinações x Número de Agentes 40

Figura 2-8 - Custo Incremental Causado pelo Agente i 42

Figura 4-1 - Níveis Hierárquicos de Confiabilidade 68

Figura 4-2 - Diagrama de Estados dos Componentes $\quad 70$

Figura 4-3 - Modelo de Markov a Dois Estados $\quad 70$

Figura 5-1 - Sistema-Exemplo de 5 Barras $\quad 91$

Figura 5-2 - Valor Unitário e Remuneração dos Geradores que Provêem o Serviço Ancilar de Suporte de Potência Reativa 96

Figura 5-3 - Diagrama Unifilar do Sistema IEEE-RTS 98

Figura 5-4 - Valor Unitário de Aumann-Shapley - Sistema IEEE-RTS 99

Figura 5-5 - Remuneração dos Geradores - Sistema IEEE-RTS 100

Figura 5-6 - Valor Unitário de Aumann-Shapley - Sistema S-SE (1) 100

Figura 5-7 - Remuneração dos Geradores - Sistema S-SE (1) 101

Figura 5-8 - Valor Unitário de Aumann-Shapley - Sistema S-SE (2) 101

Figura 5-9 - Remuneração dos Geradores - Sistema S-SE (2) 101

Figura 5-10 - Valor Unitário de Aumann-Shapley - Sistema S-SE (3) 101

Figura 5-11 - Remuneração dos Geradores - Sistema S-SE (3) 102

Figura 5-12 - Valor Unitário de Aumann-Shapley - Sistema S-SE (4) 102

Figura 5-13 - Remuneração dos Geradores - Sistema S-SE (4) 102

Figura 6-1 - Representação Esquemática da Reserva de Potência 106

Figura 6-2 - Custo de Oportunidade dos Geradores ao Prover Reserva de $\begin{array}{ll}\text { Potência } & 107\end{array}$

Figura 6-3 - Cálculo dos Limites de Geração e de Reserva de Potência 113

Figura 6-4 - Remuneração dos Geradores que Provêem o Serviço Ancilar de Reserva de Potência 120

Figura 6-5 - Valor Unitário Esperado de Aumann-Shapley - Sistema IEEE-RTS12. 
Figura 6-6 - Remuneração dos Geradores - Sistema IEEE-RTS

Figura 6-7 - Valor Unitário Esperado de Aumann-Shapley - Sistema S-SE 125

Figura 6-8 - Remuneração dos Geradores - Sistema S-SE 126

$\begin{array}{ll}\text { Figura A-1 - Caminho ABA } & 135\end{array}$ 


\section{Lista de tabelas}

Tabela 2.1 - Resultado da Repartição de Custos (milhões) 29

Tabela 2.2 - Repartição de Custos pelo Método de Custos Marginais 34

Tabela 2.3 - Repartição pelo Método de Custos Marginais com Fator de Ajuste $\phi$

Tabela 2.4 - Repartição pelo Método dos Custos Incrementais - Sequência $1-2-3$

Tabela 2.5 - Repartição pelo Método dos Custos Incrementais - Sequência 1-3-2

Tabela 2.6 - Repartição de Custos Alocado pelo Método de Shapley

Tabela 2.7 - Repartição de Custos pelo Método de Shapley - Custos Unitários39

Tabela 2.8 - Custos Alocados pelo Método de Shapley Modificado

Tabela 2.9 - Repartição pelo Método de Shapley Modificado - Custos Unitários

Tabela 2.10 - Repartição de Custos pelo Método de Aumann-Shapley

Tabela 2.11 - Vantagens e Desvantagens dos Métodos de Repartição de Custos

45

Tabela 5.1 - Parâmetros da Nova Fonte de Suporte de Potência Reativa 92

Tabela 5.2 - Custo Evitado de Instalação de Novas Fontes de Potência Reativa

Tabela 5.3 - Limites de Geração de Potência Reativa

Tabela 5.4 - Custos Marginais Associados aos Limites de Potência Reativa 94

Tabela 5.5 - Valor Unitário de Aumann-Shapley (\$/MVAr)

Tabela 5.6 - Remuneração dos Geradores

Tabela 6.1 - Limites de Potência Ativa e Reservas de Potência dos Geradores

Tabela 6.2 - Limites Ajustados de Potência Ativa

Tabela 6.3 - Custo Interrupção do Sistema-Exemplo

Tabela 6.4 - Limites de Reserva de Potência dos Geradores (MW)

Tabela 6.5 - Valor Esperado dos Custos Marginais Associados aos Limites de Reserva de Potência

Tabela 6.6 - Valor Unitário Esperado de Aumann-Shapley (\$/MW) 
Tabela A-1 - Dados de Barras AC para o Sistema-Exemplo 139

Tabela A-2 - Dados de Circuitos para o Sistema-Exemplo 139

Tabela A-3 - Dados Estocásticos de Circuitos para o Sistema-Exemplo 139

Tabela A-4 - Dados de Usinas para o Sistema-Exemplo 140

Tabela A-5 - Dados de Estocásticos de Usinas para o Sistema-Exemplo 140

Tabela A-6 - Limites de Tensão para o Sistema Exemplo 140

Tabela B-1 - Remuneração dos Geradores que Provêem o Serviço Ancilar de Suporte de Potência Reativa - Sistema IEEE-RTS 141

Tabela B-2 - Remuneração dos Geradores que Provêem o Serviço Ancilar de Suporte de Potência Reativa - Sistema Sul-Sudeste 143

Tabela C-1 -Remuneração dos Geradores que Provêem o Serviço Ancilar de Reserva de Potência - Sistema IEEE-RTS 144

Tabela C2 -Remuneração dos Geradores que Provêem o Serviço Ancilar de Reserva de Potência - Sistema Sul-Sudeste 144 